\title{
LA CONTRIBUCIÓN DE LAS ALTERACIONES PATOLÓGICAS A LA INTERPRETACIÓN EN ANTROPOLOGÍA FORENSE
}

\section{CONTRIBUTIONS OF PATHOLOGICAL ALTERATIONS TO FORENSIC ANTHROPOLOGY INTERPRETATION}

\author{
Douglas H. Ubelaker ${ }^{1}$ \\ Traducción: Claudia Mercedes Rojas-Sepúlveda ${ }^{2}$ y Astrid Lorena Perafán Ledezma²
}

\begin{abstract}
RESUMEN
La patología juega un rol principal en varios aspectos relacionados con la interpretación dentro de la antropología forense. Se presentan aquí diversas observaciones sobre los efectos de las condiciones patológicas y las formas cómo éstas pueden ser usadas para facilitar el proceso de identificación. Es justamente en el contexto de la definición más amplia de patología, como "algo anormal", que se forma en gran medida la base de la lógica, de la metodología y de la práctica de la antropología forense. De hecho, las condiciones que no son frecuentes o que no se comparten con muchas otras personas son las que pueden ser utilizadas para llegar a la identificación positiva. Los esfuerzos durante el rescate y la evaluación de la evidencia de actividad criminal (foul play), así como la estimación del sexo, la edad al momento de la muerte, la filiación poblacional, la estatura en vida, el tiempo transcurrido desde el momento de la muerte y otros componentes importantes, dependen del reconocimiento de los patrones normales y los anormales. Las múltiples contribuciones de la patología a la práctica de la antropología forense son documentadas aquí a través de casos aplicados.
\end{abstract}

Palabras clave: Patología, identificación, antropología forense

\section{ABSTRACT}

Pathology plays a key role in various aspects of interpretation within forensic anthropology. These contributions include observations of the effects of pathological conditions and using them to facilitate identification efforts. In the context of the broader definition of pathology as "something abnormal" it forms the foundation of much of the logic, methodology and practice of forensic anthropology. In fact, only unusual conditions not shared by many others can be utilized for positive identification. Recovery efforts and evaluation of the evidence of foul play, as well as the estimation sex, age at death, ancestry, living stature, time since death and other important components depend upon recognition of normal and abnormal patterns. The multifaceted contributions of pathology to the practice of forensic anthropology are firmly documented in casework applications.

Keywords: Pathology, identification, forensic anthropology

Tipología: Articulo de reflexión

Fecha de recepción: 21/01/2014

Fecha de aceptación: 18/06/2014

Como citar éste artículo: Ubelaker, D. H. (2014). La contribución de las alteraciones patológicas a la interpretación en Antropología forense. Traducción: Claudia Mercedes Rojas-Sepúlveda y Astrid Lorena Perafán Ledezma. Jangwa Pana, 13, 152 - 165

1. Department of Anthropology Smithsonian Institution. United States. E.mail: ubelaked@si.edu

2. Programa de Antropología Universidad del Magdalena. Colombia. Correo: clarosepul@gmail.com; astridunimagdalena@gmail.com 


\section{INTRODUCCIÓN}

$\mathrm{L}$ a antropología forense representa la aplicación del conocimiento y la metodología de la antropología a la resolución de asuntos médicolegales. Dichos asuntos tienen que ver con individuos vivos y aquellos recientemente muertos, pero frecuentemente se relacionan con restos humanos esqueletizados. Estas contribuciones pueden incidir en el proceso de rescate de restos humanos, en la determinación sobre si los restos materiales son o no humanos, en la estimación del sexo, de la edad al momento de la muerte, de la filiación poblacional, de la estatura en vida y del tiempo transcurrido desde el deceso. Sin embargo, las metas principales se concentran en la identificación del individuo y la evaluación de la evidencia de la posible actividad criminal (foul play). Aunque muchas series de evidencia y muchas observaciones pueden ser utilizadas, las alteraciones patológicas frecuentemente proveen información clave para la interpretación. Este ensayo explora el rol multifacético que tiene la patología en la investigación en antropología forense.

\section{Conceptos de patología}

La palabra patología se considera, de manera frecuente, como relacionada con el estudio del proceso de la enfermedad y de sus efectos. De hecho, la primera definición de patología en el diccionario Webster (Webster's 1979) dice: “el estudio de la naturaleza esencial de las enfermedades y específicamente de los cambios estructurales y funcionales producidos por ellas" (Traducción libre). Esta definición se relaciona fuertemente con el campo general de la paleopatología y con muchas aplicaciones dentro de la antropología forense. En el estudio del esqueleto humano se pueden descubrir alteraciones anormales, para cuya interpretación puede surgir la pregunta: “¿Qué enfermedad produjo estas alteraciones?". En el caso de esqueletos recuperados de contextos arqueológicos la pregunta es importante para examinar la historia, evolución e impacto de las enfermedades y explorar patrones generales de morbilidad y mortalidad en el pasado (Waldron, 2009). En el contexto forense, una respuesta precisa a la pregunta puede llevar a memorias familiares sobre la experiencia de la enfermedad, a archivos de hospital y a la identificación. Sin embargo, algunas respuestas pueden ser ambiguas ya que diferentes enfermedades pueden producir efectos similares en el esqueleto mientras que una sola enfermedad puede tener efectos múltiples y distintos (Aufderheide \& Rodriguez-Martin, 1998).

La segunda definición de "patología" en el diccionario Webster (Webster's 1979) dice simplemente: "algo anormal". Mientras que una condición anormal puede relacionarse con una enfermedad, también simplemente puede consistir en la "desviación de lo apropiado o del estado asumido como normal...". Esta condición de "anormalidad" permanece justamente en el núcleo intelectual de la antropología forense, especialmente porque se relaciona con interpretaciones sobre identificación personal y evidencia de actividad criminal (foul play). La identificación positiva depende del hallazgo de rasgos únicos e inusuales sobre los restos recuperados que puedan demostrar haber estado presentes en un individuo en particular durante su vida (Christensen \& Anderson, 2013). De manera similar, la evidencia fuerte de actividad criminal (foul play) puede ser relacionada con alteraciones anormales encontradas en restos las cuales fueron producidas por eventos cercanos $\mathrm{u}$ ocurridos en el momento mismo de la muerte (Berryman et al., 2013a,b). El concepto de anormalidad, inherente a las definiciones de patología, también enmarca entonces el núcleo de la interpretación antropológica, especialmente en lo relacionado con la identificación y la actividad criminal (foul play).

\section{Trauma perimortem}

La contribución de los antropólogos forenses a la determinación de la causa y la manera de muerte frecuentemente tiene que ver con las observa- 
ciones del trauma perimortem (Loe, 2009). El término "perimortem" se emplea en referencia al tiempo en el que sucedió el evento que produjo las alteraciones observadas, significando: en o alrededor del tiempo de la muerte. Esta definición es bastante vaga y refleja el hecho de que a partir de la mera evidencia esquelética, los antropólogos no pueden definir el tiempo exacto transcurrido entre la muerte y el evento traumático. De cierto modo, el estatus de perimortem representa una categoría por defecto: las alteraciones que carecen de evidencia de remodelación antemortem/nueva formación de hueso y aquellas que no presentan indicaciones diagnósticas de estatus postmortem. Los indicadores postmortem más comunes incluyen contrastes en la coloración entre las superficies rotas y aquellas no rotas, marcas de herramientas producidas durante los procedimientos de recuperación o rescate, alteraciones diagnósticas por dientes de roedores y de otros mamíferos, así como alteraciones producidas por el crecimiento de las raíces de las plantas o por exposición al sol (Haglund \& Sorg, 1997; Nawrocki, 2009).

Adicionalmente, la evidencia del período perimortem puede incluir características que indiquen que el hueso estaba fresco cuando las alteraciones ocurrieron. Dicha evidencia se relaciona con la respuesta plástica del hueso fresco, así como la tendencia al doblamiento o encorvamiento. En una investigación para comparar los patrones de ruptura de hueso fresco frente a hueso seco, Moraitis \& Spiliopoulou (2006) encontraron que el patrón de ruptura del hueso fresco incluye ángulos obtusos o fracturas agudas. Además notaron que en hueso fresco los bordes de la fractura eran más suaves, afilados y más biselados que en hueso seco.

El término "trauma" en el contexto de la antropología forense se relaciona de manera cercana con la definición de perimortem aportada más arriba. Las alteraciones consideradas como traumáticas no incluyen la ruptura ni el desgaste del hueso asociadas con factores postmortem.
Los tipos de trauma perimortem que se observan de manera frecuente en la casuística de la antropología forense incluyen el trauma por fuerza contundente u obtusa, por objeto cortante o afilado, la lesión por proyectil o arma de fuego, el trauma por explosión y los efectos térmicos (Loe, 2009). A pesar de que estas categorías pueden superponerse, cada una presenta caracteres diagnósticos. La interpretación refleja el entendimiento de la naturaleza de los tipos de fuerza que se aplica así como las propiedades biomecánicas del hueso (Ubelaker, 1991). Todas las formas de trauma perimortem presentan alteraciones anormales y evidencia que sugiere que éstas no reflejan variación anatómica humana normal y que no fueron producidas mucho tiempo después de la muerte. La interpretación del trauma perimortem entonces requiere el reconocimiento de los patrones de alteración asociados con las categorías mencionadas más arriba, en conjunción con otra evidencia (e.g. contexto y recuperación de proyectiles). A continuación se presentan ejemplos de formas primarias de trauma perimortem observadas en la práctica de la antropología forense.

El término trauma por fuerza contundente se refiere a las lesiones perimortem que son generales en naturaleza y que no pueden relacionarse a patrones o tipos de lesión más específicos. El trauma por fuerza contundente puede resultar de la lesión asociada a golpes con objetos, choques de vehículos o caídas desde alturas considerables. $\mathrm{Su}$ correcta interpretación requiere determinar el estatus de perimortem como se discutió más arriba, así como el reconocimiento de los patrones y del empleo de los principios biomecánicos.

La Figura 1 presenta una alteración en el lado izquierdo de un cráneo la cual refleja un trauma por fuerza contundente. El área afectada es levemente empujada hacia adentro y presenta fractura en los bordes. Mientras que el área sugiere trauma por fuerza contundente perimortem, las alteraciones no revelan suficiente detalle para un diagnóstico más preciso. 


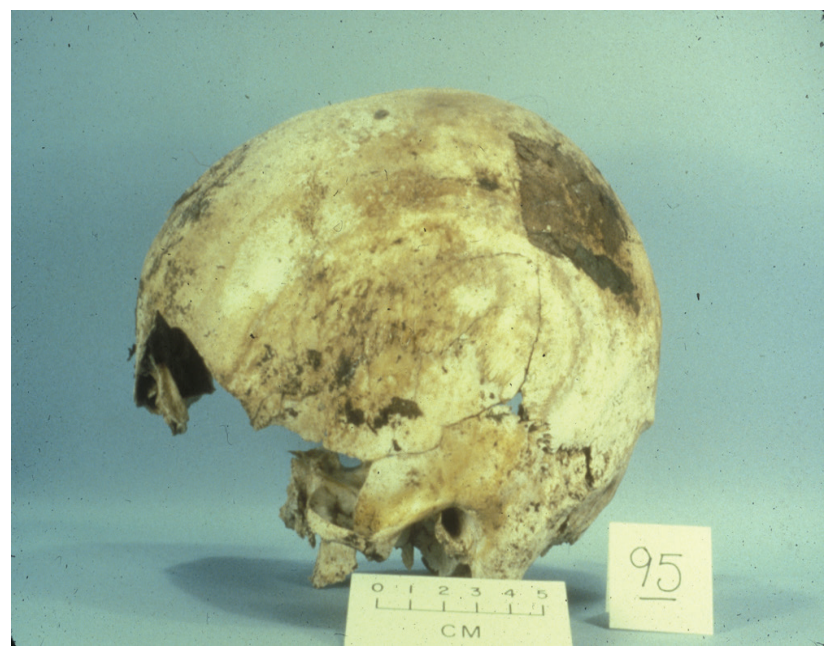

Figura 1. Alteración que refleja un trauma por fuerza contundente en el lado izquierdo del cráneo.

En algunos casos, las lesiones sufridas por trauma por fuerza contundente tienen un patrón tan claro que pueden asociarse a tipos particulares de objetos. Dicho "trauma tipo" puede sugerir características o información general sobre la naturaleza y la forma de los materiales concernientes. La medida en la cual dicho patrón pueda ser detectado depende no solamente del instrumento empleado sino también de las áreas del esqueleto impactadas.

En 1990, una disputa en un área rural en el Estado de Washington llevó a un hombre a golpear a otro con el extremo de una barra de hierro para desmontar llantas (Ubelaker \& Scammell, 1992). Aunque hubo un intento para deshacerse del cuerpo colocándolo en un lugar cercano al río, éste fue recuperado y puesto en análisis. Un examen cuidadoso reveló las alteraciones del cráneo las cuales sugirieron un impacto con un objeto duro. En particular, una de las alteraciones (Figura 2) reveló un borde exterior circular $\mathrm{y}$ un borde interior irregular. Las medidas y el patrón de esta alteración concordaban muy bien con aquellas de uno de los extremos de una barra de hierro para desmontar llantas que había sido encontrada en la escena. Mi reporte y el subsecuente testimonio en la corte señalaron las simi- litudes e indicaron que las alteraciones craneales fueron producidas por ese instrumento o por otro con las mismas características.

El trauma por fuerza cortante representa otra forma de lesión en la cual las características del tejido impactado sugieren que un instrumento filoso fue utilizado. Dichas alteraciones tienden a ser lineales con márgenes bien definidos (Ubelaker, 1999). Ya que muchos instrumentos diferentes pueden infligir trauma por fuerza cortante, la forma de las alteraciones puede variar ampliamente; muchas pueden ser bastante largas, afiladas y superficiales, mientras que otras pueden penetrar profundamente en el hueso o incluso separarlo en segmentos. La variación de la lesión puede ser marcada, si la evidencia muestra que un instrumento agudo fue utilizado.

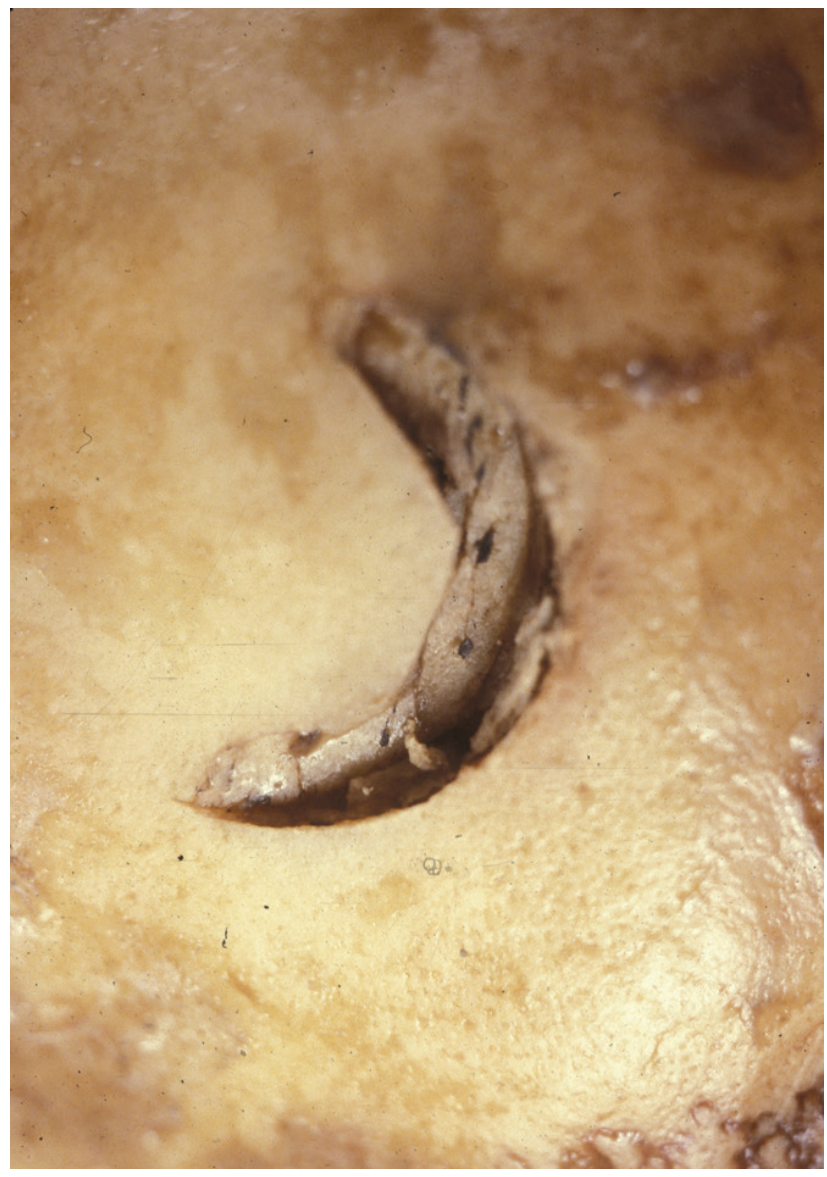

Figura 2. Alteración ósea en el cráneo con borde circular externo y borde irregular interno. 
La Figura 3 presenta un ejemplo del trauma perimortem por fuerza cortante en una costilla. Nótese la naturaleza linear de la alteración, los claros márgenes del corte y el doblamiento del segmento parcialmente separado. Si bien la naturaleza de la alteración sugiere un trauma por fuerza cortante, el doblamiento hacia afuera del segmento indica un estatus perimortem. El doblamiento hacia afuera fue posible porque el hueso estaba fresco cuando la lesión ocurrió.

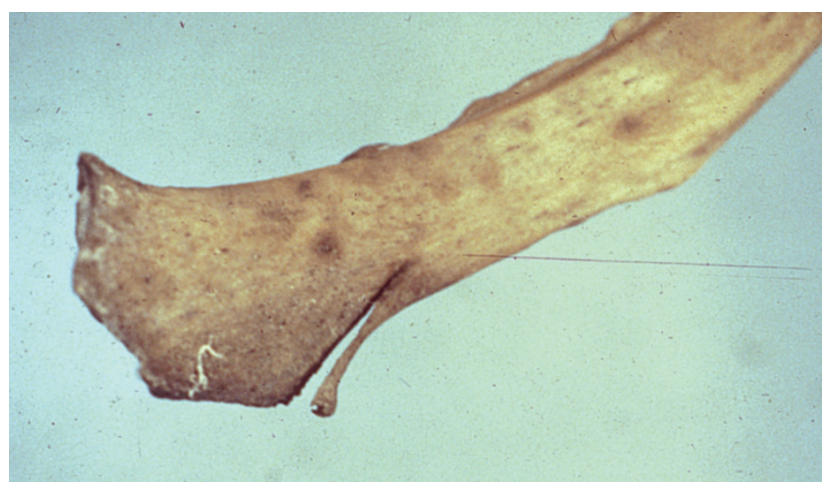

Figura 3. Trauma perimortem por fuerza cortante en costilla.

La lesión por arma de fuego puede producir alteraciones esqueléticas diagnósticas, dependiendo del hueso impactado. El impacto por proyectil en el hueso cortical denso, como el de la bóveda craneal adulta puede resultar en patrones de biselado que pueden indicar la dirección del mismo. La entrada de un proyectil de arma de fuego puede producir una perforación más ancha en la superficie endocraneal (interna) que en la superficie ectocraneal (externa) correspondiente (Figura 4). Este patrón es contrario en el sitio de salida (Figura 5). Si el proyectil golpea hueso esponjoso, el patrón de la alteración puede ser más irregular. La dirección del proyectil entonces puede ser evaluada a través de la consideración de los patrones de biselado discutidos más arriba, junto con alguna evidencia de desplazamiento del fragmento (Ubelaker, 1996). Por supuesto, la evidencia puede incluir perforaciones en prendas de vestir asociadas o incluso la misma recuperación de los proyectiles.

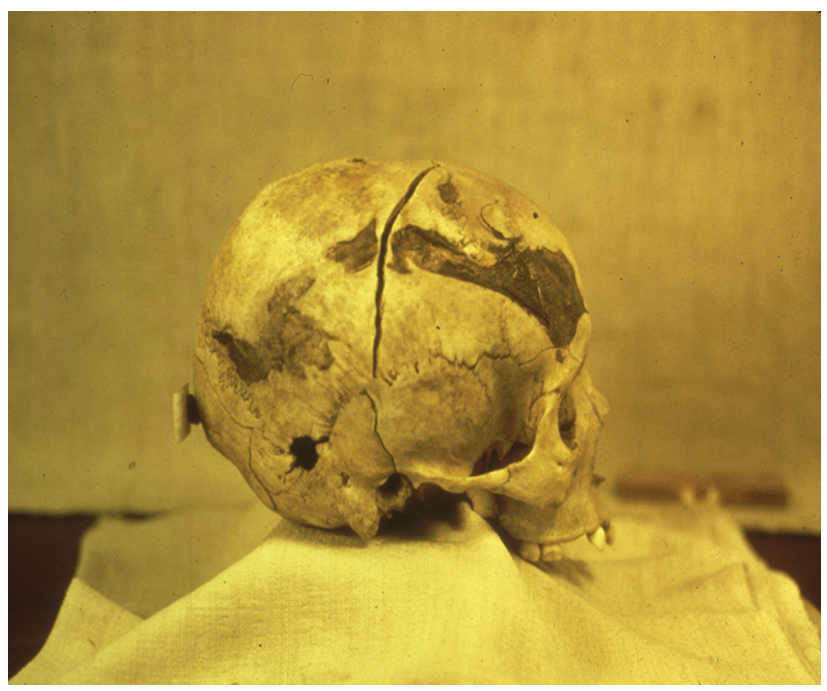

Figura 4. Entrada de un proyectil de arma de fuego.

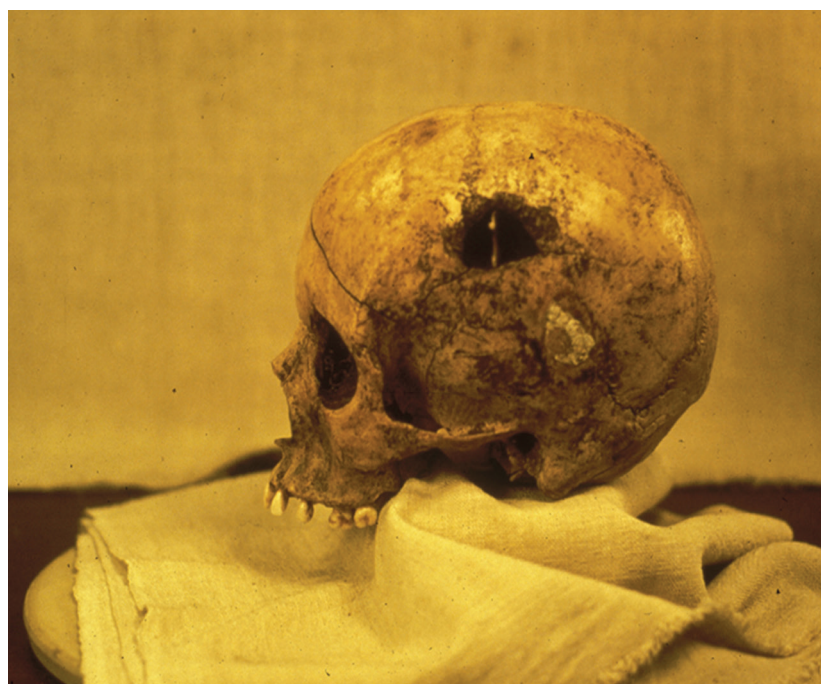

Figura 5. Salida de un proyectil de arma de fuego.

El trauma por explosión y las alteraciones térmicas pueden producir fragmentación. El trauma por explosión es frecuentemente asociado con alteraciones térmicas ya que estos tipos de trauma se relacionan de manera importante. Las alteraciones térmicas suelen presentar coloración y/o evidencias de reducción de hueso que son diagnósticas (Ubelaker, 2009). La determinación del estatus perimortem o postmortem puede ser problemática en dichos casos ya que muchos factores tafonómicos pueden llevar también a la fragmentación. Los huesos fragmentados post- 
mortem pueden también ser expuestos posteriormente a la alteración térmica.

\section{Tafonomía y pseudotrauma}

La detección e interpretación de las alteraciones patológicas en el esqueleto humano debe considerar aquellas producidas postmortem. Las alteraciones postmortem pueden ser mejor detectadas cuando producen contrastes de coloración marcados entre las superficies rotas y las intactas. Dichos contrastes ocurren cuando la ruptura es relativamente reciente, como en aquellas sufridas durante la recuperación o rescate de los restos. Una ruptura postmortem fresca de un hueso que ha sido expuesto a los elementos por muchos años, usualmente revelará el color y la textura natural del hueso en la superficie rota. Estos indicadores pueden contrastar dramáticamente con las superficies manchadas y degradas del hueso intacto.

En algunos casos, el patrón de la lesión esquelética puede sugerir trauma perimortem, pero el contraste en la coloración puede clarificar el estatus postmortem. En 1993, un trabajador de una construcción en Georgia notó, durante la preparación del sitio, la presencia de restos esqueléticos. El análisis confirmó el estatus humano de los restos y señaló una serie de fracturas a lo largo de los huesos las cuales sugerían trauma perimortem. En particular, las fracturas revelaron un patrón en "mariposa" en el cual áreas de hueso más o menos triangulares se separaron de las otras áreas de la diáfisis a causa de la fractura. Este patrón de fractura ha sido observado en trauma perimortem en el cual peatones golpeados por automóviles han sido afectados. En dichos casos, las piernas se ven comprometidas con mucha frecuencia. El sitio de impacto en el hueso sufre estrés por compresión lo cual lleva a fracturas complejas. En el lado opuesto del hueso (en la superficie que no recibe el impacto) se produce estrés por tensión el cual resulta en fracturas simples de separación del hueso.
Si bien el patrón de las fracturas en el caso de Georgia sugería trauma perimortem, como se describió más arriba, las superficies del hueso rotas claramente revelaban contrastes en la coloración. Por otro lado, las superficies exteriores que no se encontraban rotas se encontraban uniformemente manchadas debido a la exposición postmortem, mientras que las superficies rotas estaban sin manchas y revelaban el color natural del hueso (Figura 6). Aparentemente, los huesos se habían roto recientemente por el efecto de pisoteado de los equipos de energía pesada empleados en la construcción (Ubelaker \& Adams, 1995). Este efecto de pisoteo reciente simuló una bien conocida condición de trauma perimortem. El caso revela los desafíos de la interpretación forense y el valor de la cuidadosa observación de los indicadores tafonómicos.

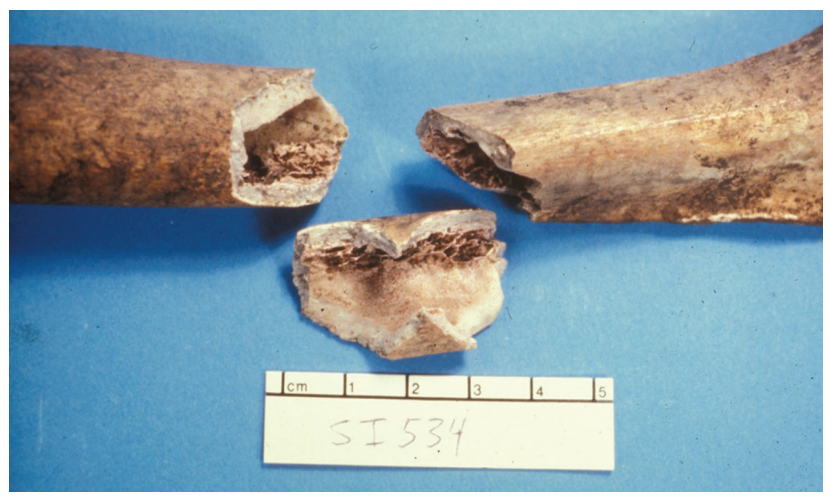

Figura 6. Fractura con patrón de mariposa, típica en accidentes automovilísticos, sin embargo, el cambio en la coloración revela que los huesos se rompieron recientemente.

Otro ejemplo es reportado por Ubelaker \& Sperber (1988) en su informe sobre un caso forense en Omaha, Nebraska. Una mujer de 19 años de edad fue reportada desaparecida en 1975, la última vez que se le vio se encontraba en un local frecuentado por la organización de motociclistas Hell's Angels (Ángeles del Infierno). En 1984, un esqueleto humano fue descubierto dentro de una cisterna fuera de uso en el aeropuerto local y fue posteriormente identificada como la mujer des- 
aparecida. Los análisis confirmaron la identidad gracias a los archivos dentales y señalaron unas alteraciones perimortem poco usuales en los huesos anteriores del cráneo las cuales sugerían la aplicación de un agente corrosivo. Estas alteraciones eran considerables en el seno frontal y en el esmalte dental afectando la dentición anterior tanto maxilar como mandibular (Figure 7).

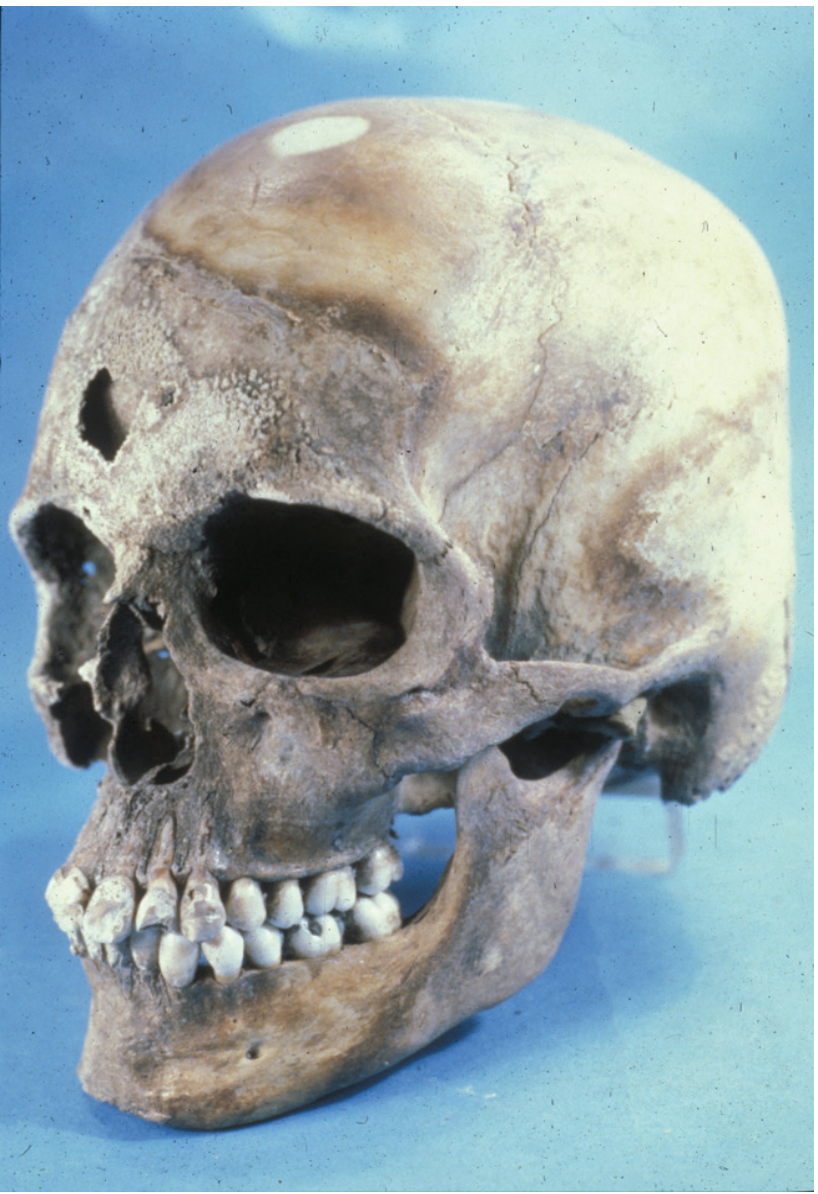

Figura 7. Alteraciones en el seno frontal y en el esmalte de la cara anterior de los dientes tanto del maxilar como de la mandíbula.

Los análisis del caso de Nebraska también señalaron alteraciones circulares en el aspecto superior del cráneo (Figura 8). Estas alteraciones eran similares en cuanto a la coloración de las áreas de destrucción corrosiva en el área facial, pero no presentaban evidencia de destrucción ósea. La interpretación final sugirió que las alteracio- nes circulares habían sido formadas postmortem a causa de la exposición solar por largos periodos de tiempo a través de perforaciones circulares de la tapa de la cisterna. A pesar de que las alteraciones circulares eran similares en la coloración a las que se produjeron en el área facial, fueron claramente postmortem y no relacionadas.

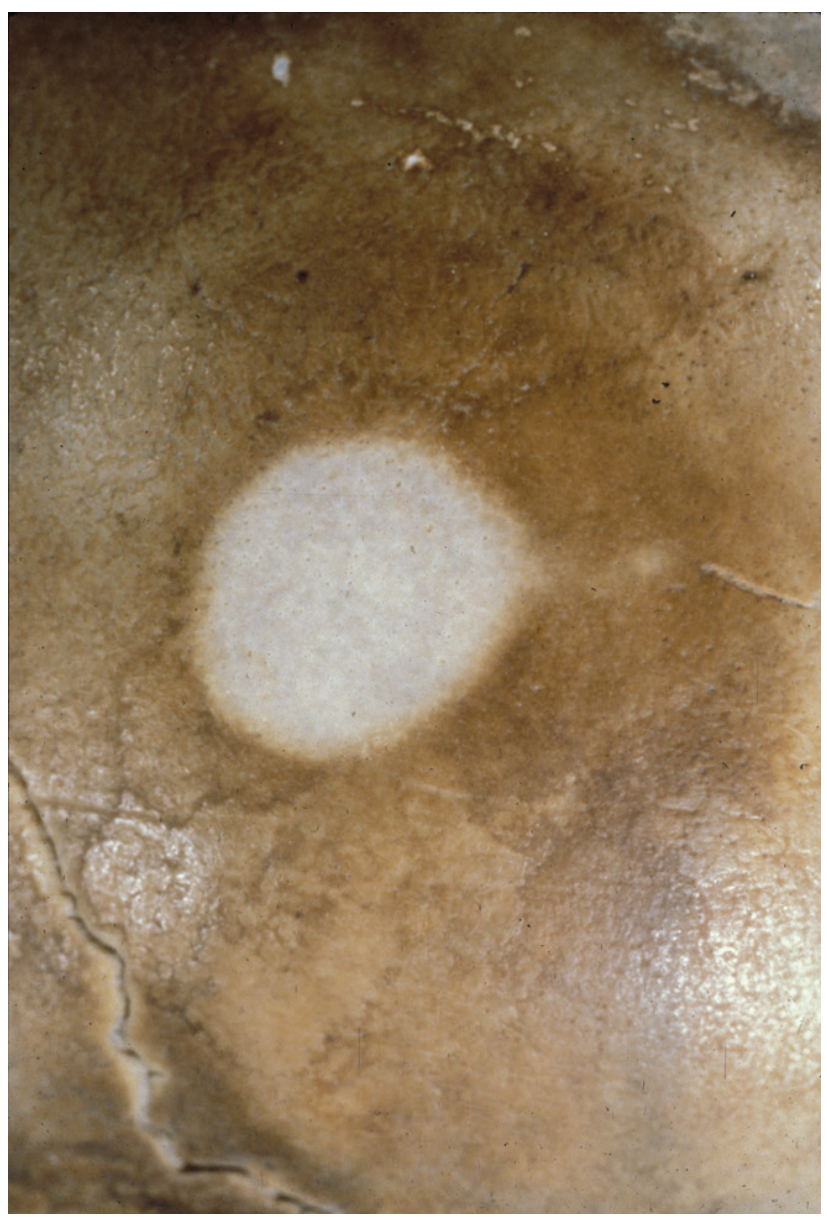

Figura 8. Alteraciones circulares en la tabla superior del cráneo.

Muchas alteraciones tafonómicas representan factores postmortem bien conocidos y fácilmente reconocibles. Las marcas de dientes de roedores u otros mamíferos pueden producir alteraciones diagnósticas del hueso. El crecimiento de las raíces de las plantas puede producir fractura de los huesos y dejar marcas características en las superficies de los huesos. La exposición al sol, el crecimiento de hongos y de algas, las manchas 
por hojas en descomposición, el contacto con el suelo y la interacción con artefactos metálicos asociados pueden representar factores tafonómicos bien conocidos (Nawrocki, 2009). Muchas de las alteraciones producidas por esos agentes pueden simular condiciones perimortem, por lo cual es necesaria la interpretación experimentada.

\section{Variación normal y anormal}

Una importante habilidad que el antropólogo forense trae al trabajo en casuística es el conocimiento de la variación anatómica normal. Dicha información es vital para reconocer los atributos anormales y evitar confundirlos con rasgos normales. La variación normal consiste en el desarrollo de detalles anatómicos que son conocidos por representar la variación humana natural. Dentro de dicha variación, los rasgos anatómicos pueden ser asociados con una ocurrencia general en diferentes poblaciones.

Cuando los rasgos están por fuera de la variación normal o son conocidos por ser raros, adquieren un valor especial en el análisis forense. Dichos rasgos inusuales pueden proveer información clave que contribuya a la identificación. Algunos aspectos de la anatomía son conocidos por exhibir gran variación en la expresión morfológica, puede ser tan grande que cada ejemplo individual ofrece información potencial para la identificación. La estructura del seno frontal representa uno de estos aspectos, pues es conocida por exhibir dicha variación, incluso, se ha comprobado que gemelos idénticos presentan algunas diferencias (Ubelaker, 1984). Por lo tanto, esta área anatómica es útil en los esfuerzos para hallar una identificación personal positiva. Otras áreas de la anatomía ósea ofrecen un potencial similar si los restos importantes para ello son recuperados y las radiografías antemortem correspondientes se encuentran disponibles.

La cuestión de la singularidad es lo más importante dentro de la ciencia forense. Dentro de la antropología forense, este asunto representa un rasgo dominante de la identificación personal. El proceso de identificación se realiza en dos pasos. En el primer paso, se debe determinar si los rasgos anatómicos en cuestión son compartidos por los restos recuperados y por la información antemortem documentada de la persona desaparecida. Estos rasgos usualmente consisten en rasgos anatómicos, como el seno frontal, discutido más arriba. La información antemortem con frecuencia se trata de radiografías del individuo tomadas antes de su muerte.

El segundo paso crítico implica la evaluación de la singularidad del rasgo compartido. Para apoyar una identificación positiva, los rasgos deben ser únicos o ser conocidos por ser compartidos con tan pocos individuos que la posibilidad de que los restos se parezcan a los de otra persona sea muy remota. Con frecuencia, dicha evaluación se apoya en un conjunto de rasgos que colectivamente son juzgados como únicos.

Por ejemplo, la técnica de la superposición fotográfica implica juzgar la relación entre los rasgos anatómicos de un cráneo recuperado con fotografías faciales antemortem de una persona desaparecida (Ubelaker, 1994). El moderno equipo disponible hoy en día para facilitar esta comparación utiliza imágenes por computador y representa un proceso sofisticado e impresionante. En mi experiencia, esta técnica es muy útil con fines de exclusión. Cuando se hallan las similitudes y la persona desaparecida no puede ser excluida, frecuentemente el informe indica que los restos pueden representar (no puede excluir) a la persona desaparecida, sin embargo, la identificación positiva no puede ser alcanzada usando esta técnica. Si bien la identificación positiva es teóricamente posible, en la realidad nunca ha sido posible aplicar esta técnica en un caso forense para producir una identificación positiva. Básicamente, no puede ser demostrado que los rasgos compartidos, tan impresionantes desde el punto de vista visual como lo son, sean realmente únicos. 
En contraste, una identificación positiva puede ser lograda cuando unos rasgos únicos son visibles en radiografías antemortem. Para ilustrarlo está el caso de un fragmento craneal recuperado en una investigación de homicidio en New England el cual se pensó representaba una persona desaparecida en particular. El trabajo cuidadoso de detective descubrió que poco tiempo antes de que muriera, la joven entró en una clínica de salud local buscando mejorar un severo dolor de cabeza. Los oficiales tomaron entonces de la clínica las radiografías craneales que estaban disponibles para comparación. Las radiografías tomadas de los restos recuperados revelaron compartir la misma morfología del seno frontal, el área de la silla turca del esfenoides y otros rasgos que eran, en mi opinión, en conjunto únicos, permitieron la identificación positiva (Ubelaker, 1984).

En otro caso, la identificación positiva se logró a través de la comparación de radiografías de tórax con restos recuperados. En 1984, los restos en gran parte esqueletizados de un hombre adulto fueron recuperados en una Reserva Indígena en Dakota del Sur. Al realizar el análisis antropológico del esqueleto, las autoridades sugirieron que los restos podrían relacionarse con una persona desaparecida. Aunque la dentición presentaba numerosas restauraciones, los esfuerzos por localizar archivos dentales antemortem fueron en vano. La búsqueda, sin embargo, reveló radiografías de tórax antemortem, las cuales fueron puestas a disposición para la comparación. Los restos recuperados, comparados con los detalles óseos visibles en las radiografías resultaron en una identificación positiva (Ubelaker, 1990).

Otro ejemplo muestra cómo pueden ser usados algunos rasgos óseos únicos revelados en las radiografías para identificar positivamente individuos vivos. Este caso se relaciona con fraudes realizados por una organización terrorista en los Estados Unidos, la cual para financiarse buscaba compensaciones a trabajadores. La investigación reveló que miembros de la organización con padecimientos pre-existentes aceptaban trabajos y luego fingían lesiones con el fin de acceder a las compensaciones para trabajadores. La investigación también reveló que algunos de esos miembros cambiaban luego sus identidades y repetían el proceso, recibiendo múltiples compensaciones por la misma lesión (Fenger et al. 1996). Cuando estos reportaban sus lesiones, en algunos casos se tomaban radiografías. Dichas radiografías, supuestamente representando diferentes individuos, fueron puestas a disposición para ser comparadas; el análisis reveló que en realidad representaban al mismo individuo. Las radiografías se enfocaban en el área del cuerpo relacionada con la lesión establecida, sobre la cual, suficientes detalles óseos pudieron ser vistos para facilitar la comparación y apoyar la identificación positiva.

\section{Enfermedad y tratamiento de la enfermedad}

Las observaciones sobre enfermedades en restos humanos contribuyen de maneras múltiples a la investigación forense. La evidencia de que un individuo sufrió de una enfermedad puede llevar a recuerdos familiares del evento o a archivos médicos. En particular los archivos médicos pueden incluir radiografías u otra evidencia que pueda contribuir a la identificación positiva. Varios de los casos discutidos más arriba son ejemplos de ello. Los eventos pasados de trauma que hayan producido fracturas en los huesos son particularmente útiles ya que usualmente requieren tratamiento médico y dicho tratamiento suele requerir la toma de radiografías. El estudio radiográfico de las lesiones antemortem y las fracturas sobre los restos recuperados pueden ser generalmente datadas (tiempo transcurrido entre el evento y la muerte) a través de la extensión de la remodelación ósea (Ubelaker \& Montaperto, 2011). Dicha evaluación debe considerar la localización de la lesión y la edad del individuo pero puede proveer información muy útil. La interpretación del tiempo del trauma antemortem puede ser especialmente invaluable en los casos de abuso infantil y puede ser usado para documentar la historia del abuso. 
El diagnóstico de la enfermedad únicamente a partir de la evidencia ósea puede ser todo un desafío ya que diferentes enfermedades pueden producir respuestas óseas similares. Aunque las lesiones traumáticas pueden ser obvias, si la muerte ocurre pronto después del evento, pueden ser difíciles de detectar; y también pueden ser difíciles de diagnosticar apropiadamente después de que se ha producido una remodelación ósea considerable. Las enfermedades sistémicas pueden ser más problemáticas, los problemas nutricionales y las infecciones localizadas también ya que sus efectos en el hueso pueden ser múltiples y pueden superponerse. El tratamiento detallado de estos asuntos es demasiado complejo para ser resumido aquí y es adecuadamente revisado en textos generales de paleopatología (e.g. Aufderheide \& Rodriguez-Martin, 1998). Aquellos que trabajan en el campo de la ciencia forense deben recordar que es necesario evitar la especulación en relación con entidades relacionadas con enfermedades que puedan llevar a conclusiones erróneas al ser poco precisas, el diagnóstico incorrecto puede despistar a las autoridades y hacer perder el rastro a la investigación. Como en los estudios de paleopatología, la descripción precisa y detallada, acompañada de interpretaciones cuidadosas, es lo más importante. Mientras que los peligros del diagnóstico preciso de la enfermedad a través únicamente de la evidencia morfológica ósea son conocidos, alguna esperanza tanto para la paleopatología como para las aplicaciones forenses surge de la reciente investigación molecular. A través de dicha investigación puede ser posible recuperar e identificar residuos de ADN del microorganismo que inició la enfermedad en restos forenses. Esta nueva aproximación al diagnóstico de la enfermedad en los restos óseos ofrece el potencial para determinar con seguridad la condición patológica sufrida por el individuo y facilitar una búsqueda mucho más enfocada de archivos y otros materiales que lleven a la identificación.

Un ejemplo del uso forense del diagnóstico molecular de la enfermedad se presenta en un caso de aplicación del suroeste de los Estados Unidos (Donoghue et al., 1999). Tras recuperar un esqueleto humano en un contexto potencialmente forense, los análisis sugirieron que representaba una joven mujer cuyo tiempo desde su muerte no se pudo determinar. Los análisis también revelaron lesiones erosivas y reactivas en el esqueleto, especialmente en las superficies viscerales de las costillas. Las lesiones sugerían tuberculosis, aunque otras enfermedades no podían ser descartadas. Para clarificar qué agente patógeno era el responsable de las lesiones y para contribuir a la investigación, una muestra de costilla con alteración patológica fue analizada por PCR. El análisis molecular reveló ADN residual de Mycobacterium tuberculosis, prueba de la enfermedad que afectó a la joven. El análisis molecular para el diagnóstico de la enfermedad representa una aproximación interesante que promete aportar información más precisa a la investigación forense.

El tratamiento médico de las condiciones patológicas, especialmente de lesiones traumáticas puede incluir inserción quirúrgica de aparatos ortopédicos. Cuando dichos aparatos se recuperan en asociación con restos humanos ofrecen información potencial no solamente sobre la cirugía, sino también sobre la identificación de la persona (Ubelaker \& Jacobs, 1995). Dichos aparatos incluyen válvulas de corazón artificiales, lentes implantados intraocularmente, aparatos de fijación, articulaciones artificiales, marcapasos, venas artificiales, tubos de ventilación del oído, implantes dentales, implantes de silicona y bombas de infusión (Praemer et al., 1992). Muchos de estos aparatos (dependiendo de su tamaño) pueden estar marcados individualmente, permitiendo de esta manera rastrear no solo el fabricante sino también el paciente. Tanto fabricantes como distribuidores pueden tener archivos que pueden ser consultados una vez se contacten. En fin, estos aparatos son comúnmente encontrados en individuos recuperados en contextos forenses, por esta razón, deben ser considerados como una fuente valiosa de información para la identificación. 
La presencia de un aparato ortopédico no necesariamente indica estatus humano. En un caso en Alaska, se halló un fragmento de diáfisis de hueso largo que exhibía una pseudoartrosis y una placa de fijación quirúrgicamente implantada (Ubelaker, 1999). La presencia de hueso remodelado sobre algunas porciones de la placa indicaba no solamente que representaba una condición antemortem, sino que la cirugía había tenido lugar mucho tiempo antes de la muerte. Los animales habían roído los dos extremos del hueso destruyendo así detalles anatómicos de la metáfisis y de las epífisis.

Las radiografías revelaron una pérdida cortical considerable (ensanchamiento del hueso a ambos extremos) sugiriendo una reducción mínima de la longitud como resultado del ataque de los animales. Esta observación llevó a la decisión de remover una muestra para realizar una sección histológica. El análisis microscópico de la delgada sección reveló un patrón de bandas de osteones (Mulhern \& Ubelaker, 2001), común en muchos animales no humanos y ausente en humanos. Aparentemente, el hueso provenía de un perro de gran tamaño al cual, tras haberse fracturado una pierna, se le practicó una cirugía veterinaria para estabilizar el área. La investigación reveló que los cirujanos veterinarios que operan animales grandes frecuentemente usan los mismos aparatos ortopédicos que se usan en humanos.

Las condiciones patológicas pueden también confundir en la detección del estatus no animal de la morfología del hueso. En 1988, dos calvarias encontradas en Oklahoma fueron sometidas a análisis (Ubelaker et al., 1991). Ambas habían sido roídas por animales y no presentaban área facial. Una fontanela bregmática muy grande estaba presente lo cual indicaba un estatus inmaduro. La forma redondeada y el tamaño sugerían un estatus humano o probablemente un animal grande con bóveda craneana redondeada. El análisis cuidadoso reveló un foramen magno en posición posterior, depresiones parietales y otras caracte- rísticas que los sacaban de un estatus humano, pero la morfología tampoco concordaba con la de ningún mamífero aparentemente normal.

Dos series de evidencia sugirieron un origen bovino de los especímenes. El análisis de pelo asociado reveló una concentración de cuerpos ovoides característicos de los bovinos. Adicionalmente, se examinó tejido blando asociado usando evaluación inmunológica para la cual se emplearon anticuerpos específicos. Con uno de los especímenes una línea de precipitación se formó cuando se expuso a suero antibovino sugiriendo un origen bovino del espécimen. Con esta información, una búsqueda de la literatura veterinaria y una consulta con colegas reveló que los especímenes representaban cráneos que habían sufrido hidrocefalia. Esta condición implica un incremento en el volumen del fluido cerebroespinal y la distención de los ventrículos cerebrales. Cuando esto ocurre en un animal en crecimiento, puede llevar a un agrandamiento de la bóveda craneana. El cráneo normal de un ternero puede ser fácilmente diferenciable de uno de humano por su forma alargada, además de otras características; sin embargo, con hidrocefalia el alargamiento de la bóveda craneal puede simular la morfología humana y puede llevar a confusión en ausencia de estructura facial ósea.

\section{Impactos de la patología en la metodología}

Las condiciones patológicas y otras formas de anormalidad pueden impactar otras áreas del análisis antropológico forense. La mayoría de los métodos aplicados en antropología forense se originan en bases de datos con rangos establecidos de variación. En muchos de los estudios realizados al respecto los investigadores excluyen especímenes con obvias o conocidas condiciones patológicas. Si bien la historia de la enfermedad pudo haber llevado a excluir de la investigación original a algún espécimen, puede que la misma 
no sea visible en un cierto caso forense. Esto quiere decir que potencialmente un método desarrollado a partir de una base de datos que hubiera excluido individuos con problemas de salud en particular puede ser aplicado a un esqueleto forense aun cuando tuviera en su historia dichos problemas.

La anormalidad individual puede también llevar a una interpretación inapropiada de las probabilidades. Por ejemplo, un individuo anormal puede presentar información que lo ubica, sin que se pueda detectar, en los extremos del rango de variación, un informe de ello podría entonces relacionar las probabilidades asociadas con el método en general cuando el error real en relación con el individuo particular podría ser considerable. Los asuntos de este tipo, de representación de morfología inusual, pueden relacionarse con los métodos de estimación de edad al momento de la muerte, del sexo, de la estatura en vida, de la filiación poblacional, del tiempo desde la muerte y otras áreas de análisis.

\section{Resumen y conclusiones}

El reconocimiento y la interpretación de condiciones patológicas y otras formas de variación anormal constituyen un componente crítico de la práctica en antropología forense. La detección precisa y el diagnóstico adecuado de la enfermedad pueden colaborar directamente en la determinación de la causa y la manera de muerte y proveen información que puede llevar a la identificación positiva. Este diagnóstico diferencial requiere no solamente del entendimiento del proceso de la enfermedad y de sus efectos en el hueso, sino también del conocimiento de los rasgos antemortem y postmortem que pueden simular las condiciones patológicas.

Si se considera la definición más general de patología como anormalidad, su impacto en antropología forense es enorme. El concepto de anormalidad o de condiciones inusuales forma la base de la identificación positiva y alimenta las interpretaciones de la evidencia perimortem de actividad criminal (foul play). La identificación positiva depende del descubrimiento de rasgos únicos que sean compartidos entre los restos recuperados y las características antemortem conocidas de la persona desaparecida. Para ser útiles en la identificación positiva, dichos rasgos debe ser entonces anormales y no compartidos por otros individuos.

La evidencia de actividad criminal (foul play) trae también el reconocimiento de los rasgos anormales que pueden ser relacionados con el tiempo perimortem. El trauma por fuerza contundente, la lesión por arma de fuego, el trauma por fuerza cortante, el trauma por explosión y la lesión térmica, representan condiciones anormales que se ubican dentro de las definiciones generales de patología.

El entrenamiento en antropología forense debe incluir bases en patología y en variación humana. Muchos de los problemas más generalizados en la práctica de la antropología forense se relacionan de varias maneras con la interpretación de la patología. Es solamente a través del completo entendimiento de la variación humana que las condiciones patológicas pueden ser reconocidas e integradas dentro de la interpretación.

\section{REFERENCIAS BIBLIOGRÁFICAS}

Aufderheide, A.C. \& Rodriquez-Martin, C. (1998). Human Paleopathology. Melbourne, Australia: Cambridge University press.

Berryman, H.E., Shirley, N.R. \& Lanfear, A.K. (2013a). Low-Velocity Trauma. En: M.A. Tersigni-Tarrant \& N.R. Shirley (Eds.) Forensic Anthropology: An Introduction (pp. 271-290). Boca Raton, FL: CRC Press.

Berryman, H.E., Shirley, N.R. \& Lanfear, A.K. (2013b). Basic Gunshot Trauma Interpretation in Forensic Anthropology. En: M.A. TersigniTarrant \& N.R. Shirley (Eds.) Forensic Anthropology: An Introduction (pp. 291-306). Boca Raton, FL: CRC Press. 
Christensen, A.M. \& Anderson, B.E. (2013). Methods of Personal Identification. En: M.A. Tersigni Tarrant \& N.R. Shirley (Eds.) Forensic Anthropology: An Introduction (pp. 397-420). Boca Raton, FL: CRC Press.

Donoghue, H.D. Ubelaker, D.H. \& Spigelman, M. (1999). The Use of Paleomicrobiological Techniques in a Current Forensic Case. En: G. Pálfi, O. Dutour, J. Deák \& I. Hutás (Eds.), Tuberculosis Past and Present (pp. 363-368). Szeged, Hungary: Golden Book Publisher, Ltd., Tuberculosis Foundation.

Fenger, S.S., Ubelaker, D.H. \& Rubinstein, D. (1996). Identification of Workers' Compensation Fraud Through Radiographic Comparison. Journal of Forensic Identification, 46(4), 418-431.

Haglund, W.D. \& Sorg, M.H. (Eds.). (1997). Forensic taphonomy. Boca Raton, FL: CRC Press. Loe, L. (2009). Perimortem Trauma. En: S. Blau \& D.H. Ubelaker (Eds.) Handbook of Forensic Anthropology and Archaeology (pp. 263-283). Walnut Creek, California: Left Coast Press.

Moraitis, K. \& Spiliopoulou, C. (2006). Identification and Differential Diagnosis of Perimortem Blunt Force Trauma in Tubular Long Bones. Forensic Science, Medicine, and Pathology, 2-4, 221-229.

Mulhern, D.M. \& Ubelaker, D.H. (2001). Differences in Osteon Banding Between Human and Nonhuman Bone. Journal of Forensic Sciences, 46(2), 220-222.

Nawrocki, S. P. (2009). Forensic taphonomy. En: S. Blau \& D.H. Ubelaker (Eds.) Handbook of Forensic Anthropology and Archaeology (pp. 284-294). Walnut Creek, California: Left Coast Press.

Praemer, A., Furner, S., \& Rice, D. P. (1992). Medical implants and major joint procedures. Musculoskeletal conditions in the United States (pp.125-145). Park Ridge, IL: American Academy of Orthopaedic Surgeons.

Ubelaker, D.H. (1984). Positive Identification from the Radiographic Comparison of Frontal Sinus Patterns. En T.A. Rathburn \& J. E.
Buikstra (Eds.) Human Identification Case Studies in Forensic Anthropology, (pp. 399411). Springfield: Charles C. Thomas.

Ubelaker, D.H. (1991). Perimortem and Postmortem Modification of Human Bone. Lessons from Forensic Anthropology. Anthropologie, 24 (3), 171-174.

Ubelaker, D.H. (1990). Positive Identification of American Indian Skeletal Remains from Radiographic Comparison. Journal of Forensic Sciences, 35(2), 466-472.

Ubelaker, D.H. (1994). Cranial Photographic Superimposition. En C.H. Wecht (Ed.) Forensic Sciences vol. 2, (pp. 27C1-27C39). New York: Matthew Bender and Co.

Ubelaker, D.H. (1996). The Remains of Dr. Carl Austin Weiss: Anthropological Analysis. Journal of Forensic Sciences, 41(1), 60-79. Ubelaker, D.H. (1999). Human Skeletal Remains, Excavation, Analysis, Interpretation (3 ${ }^{\text {ra }}$ ed.). Washington, D.C.: Taraxacum.

Ubelaker, D.H. (2009). The Forensic Evaluation of Burned Skeletal Remains: A Synthesis. Forensic Science International, 183, 1-5.

Ubelaker, D.H. \& Adams, B.J. (1995). Differentiation of Perimortem and Postmortem Trauma Using Taphonomic Indicators. Journal of Forensic Sciences, 40(3), 509-512.

Ubelaker, D.H., Berryman, H.E., Sutton, T.P. \& Ray, C.E. (1991). Differentation of Hydrocephalic Calf and Human Calvariae. Journal of Forensic Sciences, 36(3), 801-812. Ubelaker, D.H. \& Jacobs, C.H. (1995). Identification of Orthopedic Device Manufacturer. Journal of Forensic Sciences, 40(2), 168-170.

Ubelaker, D.H. \& Montaperto, K.M. (2011). Biomechanical and Remodeling Factors in the Interpretation of Fractures in Juveniles. In A.H. Ross \& S.M. Abel (Eds.), The Juvenile Skeleton in Forensic Abuse Investigations (pp. 33-48). New York, NY: Humana Press.

Ubelaker, D.H. \& Scammell, H. (1992). Bones, A Forensic Detective's Casebook. New York, NY: Harper Collins. 
Ubelaker, D.H. \& Sperber, N.D. (1988). Waldron, T. (2009). Paleopathology. New York, Alterations in Human Bones and Teeth as a NY: Cambridge University Press.

Result of Restricted Sun Exposure and Contact Webster's New Collegiate Dictionary. (1979). with Corrosive Agents. Journal of Forensic Sciences, 33(2), 540-548. 